\title{
Developed Distributed Energy-Efficient Clustering (DDEEC) Algorithm based on Fuzzy Logic Approach for Optimizing Energy Management in Heterogeneous WSNs
}

\author{
Anu Rathee \\ Assistant Professor \\ Institute of Marketing \\ Management
}

\author{
Indu Kashyap \\ Associate Professor \\ Computer Science Department \\ Manav Rachna International \\ University
}

\author{
Kavita Choudhary \\ Associate Professor \\ Computer Science Department \\ Jagannath University
}

\begin{abstract}
Energy Management can be improved by proficient clustering algorithms in heterogeneous wireless sensor networks. Coordination through cluster head selection provides efficient data aggregation that reduces communication overhead in the network. In this paper, we propose a fuzzy logic approach based DDEEC clustering algorithm which aims to prolong the lifetime of nodes in heterogeneous WSNs. We compare this algorithm with the PSO based DDEEC algorithm and original DDEEC algorithm according to the parameters of first node dies at different rounds and energy-efficiency metrics. The efficiency of proposed optimized fuzzy algorithm is proved by the Matlab experimental results. Simulation results exhibits that the proposed algorithm has higher energy efficiency and can improve life span of a node and data delivery at the base station over its comparatives.
\end{abstract}

\section{Keywords}

Heterogeneous WSN; cluster head; fuzzy logic, DDEEC, Energy conservation, network lifetime.

\section{INTRODUCTION}

Wireless sensor networks is an intelligent distributed sensor system providing a wide range of applications in both military as well as civilian domains connected via wireless links. WSN have limited computational power and limited memory and battery power. The key challenge in setting up and proper operation of WSN is increase the life span of the network by optimizing the energy consumption. Usually nodes in WSN are power constrained due to limited battery, it is also not possible to recharge or replace battery of already deployed nodes and nodes might be placed where they cannot be accessed. Heterogeneous WSN comprises of different types of nodes derived from different characteristics. The functioning of the sensor nodes differs depending on the nature of the characteristics of SNs. Some nodes perform sensing operation; some performs filtering operation, while others perform fusion of data and transfer of data to the base station. All the nodes have to send their data towards BS often called as sink. Nodes may be present far away from BS so direct communication is not feasible due to limited battery as direct communication requires high energy. A sensor network can be made scalable by assembling the sensor nodes into groups i.e. clusters. Energy consumption for aggregation of data is much less as compared to energy used in data transmission. In Data aggregation, fewer messages are sent to BS and only few sensor nodes have to transmit over large distance, so high energy is saved and over all lifetime of the network is prolonged. The life span of the network is extended in terms of rounds which is the process of gathering all the data from sensor nodes to the base station regardless of how much time it makes. Low-Energy Adaptive Clustering Hierarchy (LEACH) , Power Efficient Gathering in Sensor Information Systems (PEGASIS) [3], Hybrid Energy-Efficient Distributed clustering (HEED) [4] are algorithms designed for homogenous WSN under consideration so these protocols do not work efficiently under heterogeneous scenarios because these algorithms are unable to treat nodes differently in terms of their energy. Whereas, Stable Election Protocol (SEP) [5], Distributed Energy-Efficient Clustering (DEEC) [6], Developed DEEC (DDEEC) [7], Enhanced DEEC (EDEEC) [8] are algorithms designed for heterogeneous WSN. SEP is designed for two level heterogeneous networks, so it cannot work efficiently in three or multilevel heterogeneous network. SEP considered only normal and advanced nodes where normal nodes have low energy level and advanced nodes have high energy. DEEC, DDEEC, EDEEC and TDEEC are designed for multilevel heterogeneous networks and can also perform efficiently in two level heterogeneous scenarios.

In this paper, we proposed a fuzzy logic based approach in Developed Distributed Energy-Efficient Clustering to optimize the energy consumption in heterogeneous WSNs. Fuzzy logic is utilized for handling the uncertainties in cluster-head election procedure. Fuzzy logic approach has potential for dealing with conflicting situations and imprecision in data using heuristic human reasoning without needing complex mathematical modeling.

\section{RELATED WORK}

Qing et al [6] proposed a distributed energy efficient clustering (DEEC) scheme for heterogeneous WSNs. The author considered cluster head election probability on the ratio of residual energy and the average energy of the node. At point where advanced nodes having same residual energy like normal nodes DEEC continues to punish the advanced nodes so this is not optimal way for energy distribution because by doing so, advanced nodes are continuously a $\mathrm{CH}$ and they die more quickly than normal nodes. Duan et al. [12] proposed a distributed energy balance clustering (DEBC) protocol in which cluster heads are selected by a probability depending on the basis of remaining energy of node and the average energy of network. The high initial and remaining energy nodes have more chances to be the cluster heads than the low energy nodes. 
Elbhiri et al. [14] proposed a developed distributed energy efficient clustering scheme (DDEEC) to avoid this unbalanced case of cluster head selection that makes some changes in DEEC to save advanced nodes from being punished over and again. DDEEC introduces threshold residual energy to save advanced nodes from being punished over and again. This technique was based on changing dynamically and with more efficiency the cluster head election probability. Simulation results showed that the protocol performs better than the SEP and DEEC in terms of network lifetime and first node dies. The application of Computational Intelligence (CI) in WSN can be used to resolve various challenges in this arena. Fuzzy logic is one of the most important paradigms in CI technique. Fuzzy logic can be used for real-time decisions in WSN. Different parameters can be merged according to the predefined fuzzy rules and making decision based on the output result is a vital application of fuzzy control. Typically, various clustering parameters are combined by fuzzy rules to make a decision for selection a $\mathrm{CH}$ during fuzzy-clustering in WSN.

\section{PROPOSED WORK}

\subsection{Fuzzy logic approach in DDEEC}

$\mathrm{P}$ This algorithm considered two radio models, free space model and the multi-path fading channel model used by DDEEC. When the distance between the transmitter and receiver is less than threshold value $d o$, the algorithm adopts the free space model ( $d 2$ power loss). Otherwise the algorithm adopts the multi-path fading channel model ( $d 4$ power loss). So if the transmitter sends an 1-bit message to the receiver up to a distance of $d$, the energy consumption of the transmitter and $t$ receiver can be calculated by the following equations:

$$
\operatorname{ETx}(\mathrm{k}, \mathrm{d})=\left\{\text { Eelec } * \mathrm{k}+\varepsilon f s * \mathrm{k} * \mathrm{~d}^{2}, \mathrm{~d}<\mathrm{d} 0,\right.
$$

Eelec $* \mathrm{k}+\varepsilon m p * \mathrm{k} * \mathrm{~d}^{4}, \mathrm{~d} \geq \mathrm{d} 0$

$$
\operatorname{ERx}(\mathrm{k})=\text { Eelec } * \mathrm{k}
$$

$E T \mathcal{X}(\mathrm{k}, \mathrm{d})$ is the transmitter energy used to send an k-bit message to the receiver up to a distance of $\mathrm{d} ; E R \mathcal{X}(\mathrm{k})$ is the receiver energy used to receive an k -bit message; Eelec is the energy consumption of the wireless send-receive circuit.

The most important part of the proposed algorithm is Fuzzy Inference System (FIS). The FIS has four parts and the architecture of the model is shown in Figure 1.

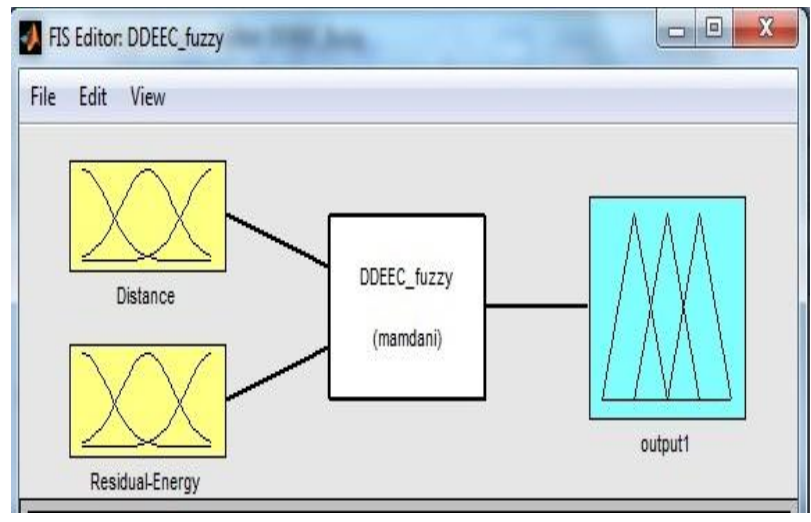

Fig. 1: FIS Architecture

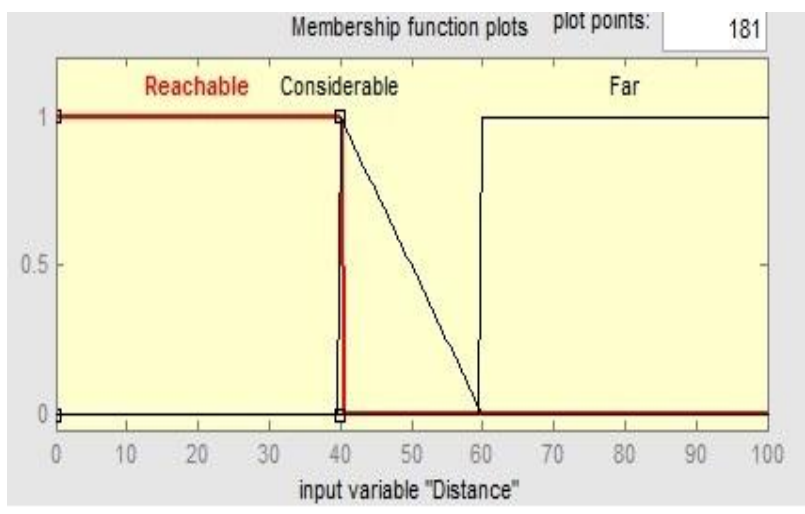

Fig. 2: Minimum distance rule base input

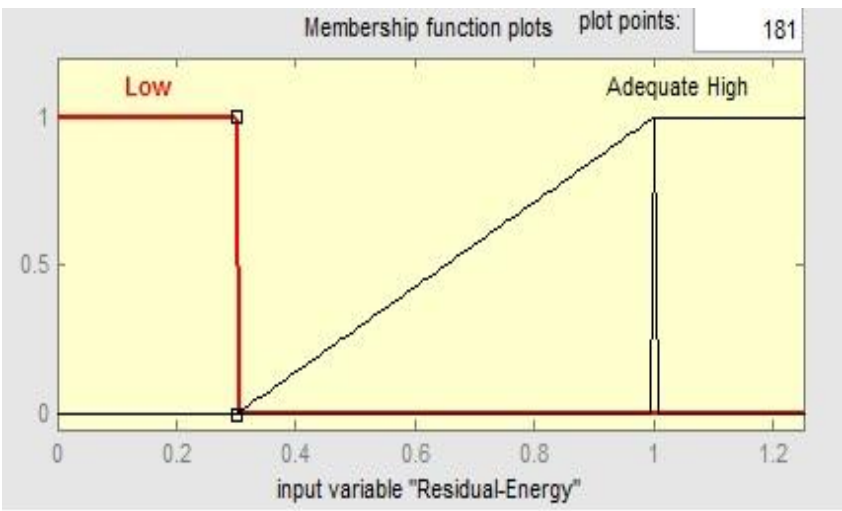

Fig. 3: Residual Energy rule base input

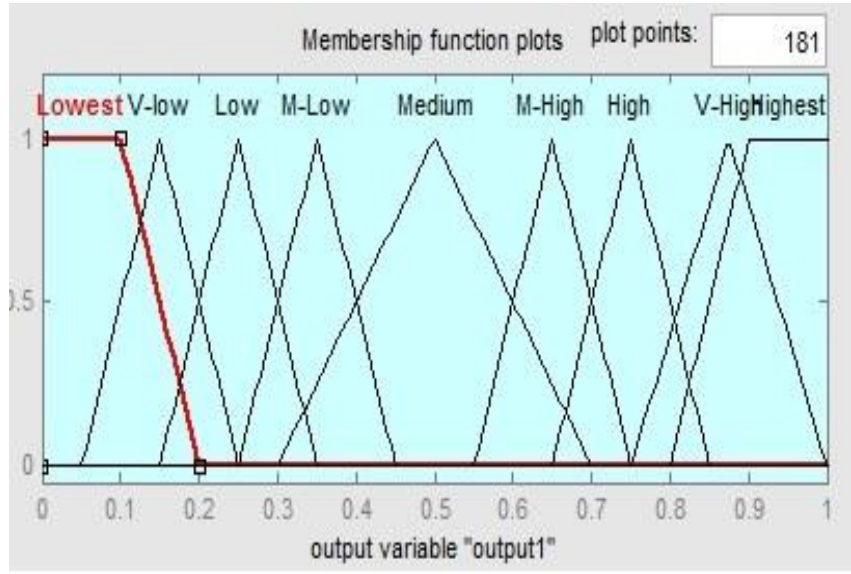

Fig. 4: Cluster head selection output variable

We use Mamdani's[6] method for the inference process of the proposed method. Mamdani technique allows us to describe the expert knowledge in a more intuitive manner; we have used it in our system. Mamdani's method is most commonly used in applications due to its simple structure of 'min-max' operations. There are four steps to get the crisp value from the FIS system. The first step is to evaluate the antecedent for each rule. The second step is to obtain each rule's conclusion. The third step is to aggregate conclusions and the last step is Defuzzification. 


\subsection{DDEEC-FL System Model}

In this paper the Cluster-heads are elected in each round by calculating the chance each node has to become the clusterhead by considering these fuzzy rule based descriptors energy level in each node and its minimum distance with respect to the base station. In this model we are having three types of sensor nodes i.e. normal nodes, advance nodes and super nodes. These nodes are differentiated according to their energy levels. DDEEC, Developed Distributed EnergyEfficient Clustering, permits to balance the cluster head selection with their residual energy. The probability of election of cluster head is set to be 0.1.Initial energy of the normal nodes is 0.5 joules.

So, the super and advanced nodes are largely solicited to be selected as cluster heads for the first transmission rounds, and when their energy decrease sensibly, these nodes will have the same cluster head election probability like the normal nodes. Threshold distance required for communication among the nodes with the cluster head and base station is depending on the parameters of free space loss and multi path loss.

\section{Preliminaries}

Threshold Distance (do)=sqrt(Efs/Emp);

$$
\begin{gathered}
\mathrm{m}=0.5 ; \\
\mathrm{mo}=0.4 ; \\
\text { normal }=\mathrm{n} *(1-\mathrm{m}) ; \\
\text { advance }=\mathrm{n} * \mathrm{~m} *(1-\mathrm{mo}) ; \\
\text { super }=\mathrm{n} * \mathrm{~m} * \mathrm{mo} ; \\
\mathrm{E}(\mathrm{t})=1 / \mathrm{N} * \mathrm{E}_{\text {total }}(1-\mathrm{r} / \mathrm{R}) \\
\mathrm{R}=\mathrm{E}_{\text {total }} / \mathrm{E}_{\text {round }} \\
\mathrm{E}_{\text {Round }}=\mathrm{L}\left(2 \mathrm{NEelec}+\mathrm{N}_{\text {eda }}+\mathrm{kEmpd}{ }_{\text {toBS }}+\mathrm{NEfsd}^{2}{ }_{\text {toCH }}\right)
\end{gathered}
$$

\section{Proposed Algorithm}

\section{Initial Round}

1. $\mathrm{CHs}$ are selected randomly and broadcast the $\mathrm{CH} \_m s g(\mathrm{CH}$ message).

2. Cluster formation and data transfer take place.

3. Each node calculates the residual energy and minimum distance from the base station and sends them to BS through $\mathrm{CH}$.

\section{End}

\section{General Rounds}

1. Output (fuzzy cost) $\leftarrow$ calculated by node using minimum distance from the BS and residual energy

2. CHs are selected based on the value of fuzzy cost and broadcast the $\mathrm{CH} \_\mathrm{msg}$.

\section{Cluster formation and data transfer take place.}

4. Each node calculates the residual energy and minimum distance and sends the values to BS through $\mathrm{CH}$

\section{End}

Table 1: Rule base for fuzzy logic

\begin{tabular}{|c|c|c|c|}
\hline Rule & Distance & Residual Energy & Output \\
\hline 1 & Reachable & Low & Low \\
\hline 2 & Reachable & Adequate & Very High \\
\hline 3 & Reachable & High & Highest \\
\hline 4 & Considerable & Low & Medium \\
\hline 5 & Considerable & Adequate & High \\
\hline 6 & Considerable & High & High \\
\hline 7 & Far & Low & Lowest \\
\hline 8 & Far & Adequate & Very low \\
\hline 9 & Far & High & Medium low \\
\hline
\end{tabular}

\section{SIMULATION MODEL}

In this paper we anticipated a Fuzzy logic based DDEEC protocol whose performance is compared with PSO based DDEEC with the Matlab Simulator, used to simulate the sensor network topology with secured protocols. In this regards Fuzzy based optimization of DDEEC algorithm utilizes distance and remaining energy level of contender node prior electing it as cluster head node. At the starting of rounds distance from distance form base station and remaining energy level of each node is calculated and stored at base station. During rounds, when a node tries to participate in cluster head election process its distance and remaining energy level is applied to fuzzy inference system which outputs cluster head election chance of a particular node. Finally the node with maximum chance value is chosen to become cluster head and starts network operation. DDEECFuzzy algorithm uses same method for cluster head rotation as used in LEACH and other clustering protocols. A cluster head node with probability $P$ cannot become cluster head during next $\left(1-\frac{1}{P}\right)$ rounds. In such way fuzzy based architecture optimizes the performance of conventional DDEEC algorithm. There are total 5000 rounds in our model as compared to the original DDEEC in which approximate all nodes are dead around 2500 rounds. In each round of the scenario, clusters-heads are selected and clusters are formed according to their high energy level. Next, each ordinary node forwards data packets to its cluster-head. Each cluster-head aggregates the received data and forwards it to the base station by calculating the minimum distance between the base station and the particular node with a particular routing protocol or directly transmits the aggregated data to the base station. In this approach we have 100 sensor nodes in which 50 nodes are normal, 30 are advanced nodes and 20 are super nodes. The network topology is made up of randomly distributed nodes in a region, in which the base station is located at the center of the area. Fig 5 shows the network diagram of the deployment model in which dark nodes are elected as cluster head for a given round. In each round, each ordinary sensor node transmits 4000 bits of data to its cluster-head. The cluster-head which receives the data from its cluster members aggregates the received data with $10 \%$ aggregation ratio. 


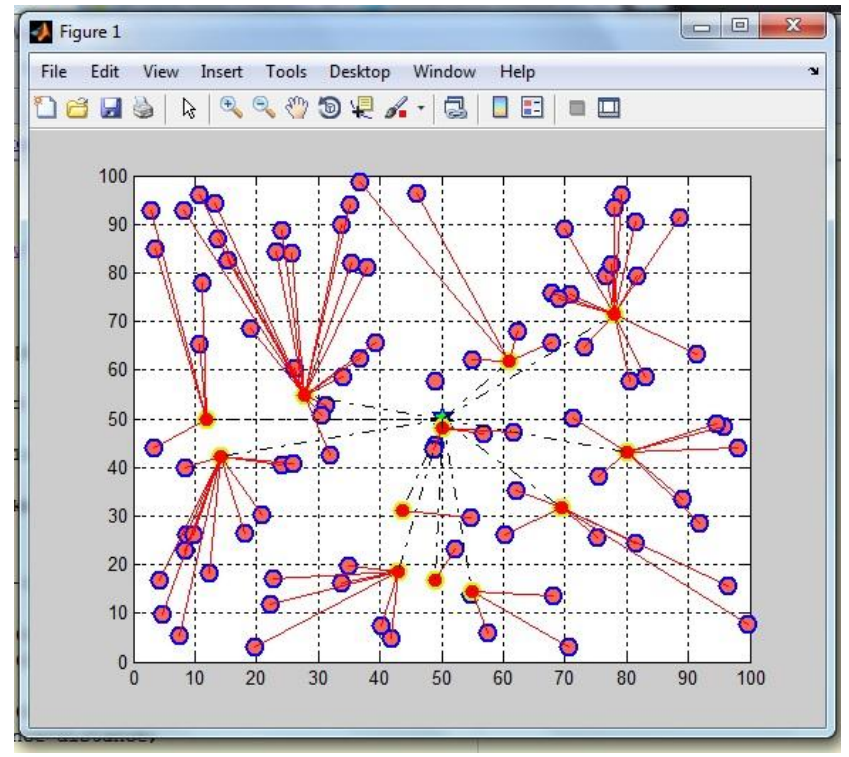

Fig 5: Fuzzy based DDEEC network of sensor nodes

\section{RESULTS AND DISCUSSIONS}

The proposed FL-DDEEC algorithm uses three level of energy heterogeneity in the network. It contains three types of nodes normal, advanced and super nodes based on their initial energy. $\boldsymbol{p}_{\boldsymbol{i}}$ Is probability used for $\mathrm{CH}$ selection and $\boldsymbol{p}_{\boldsymbol{o p t}}$ is reference for $\boldsymbol{p}_{\boldsymbol{i}}$. DDEEC uses different $\boldsymbol{p}_{\boldsymbol{o p} \boldsymbol{t}}$ values for normal, advanced and super nodes, so, value of pi in DDEEC is as follows:
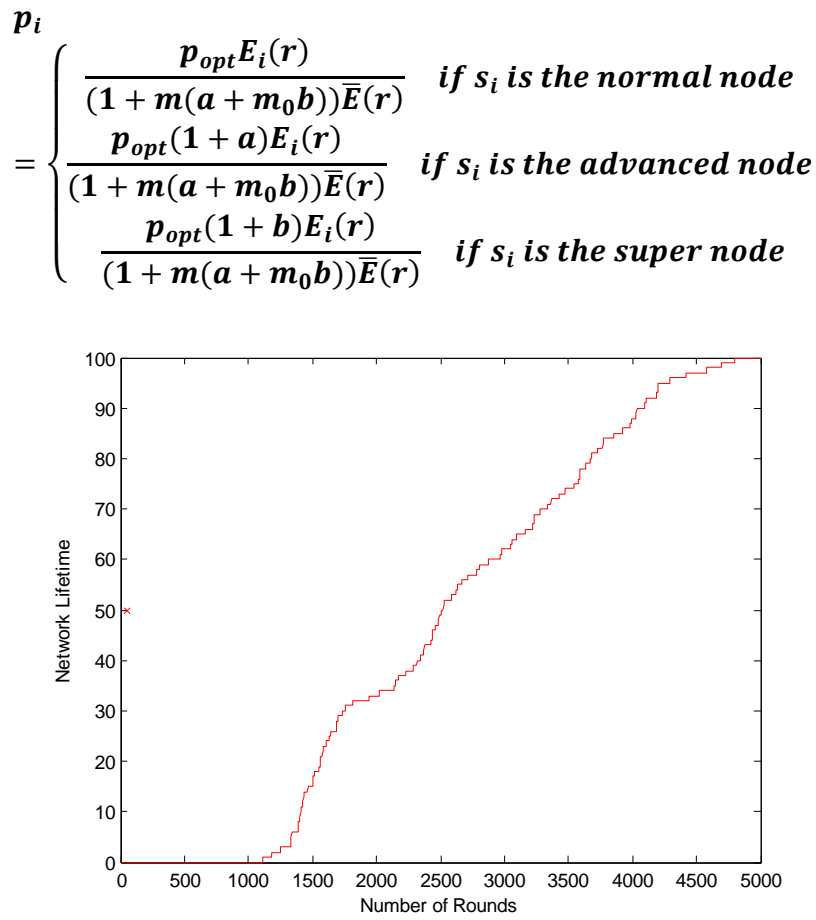

Fig6: Network lifetime of sensor nodes in PSO based DDEEC

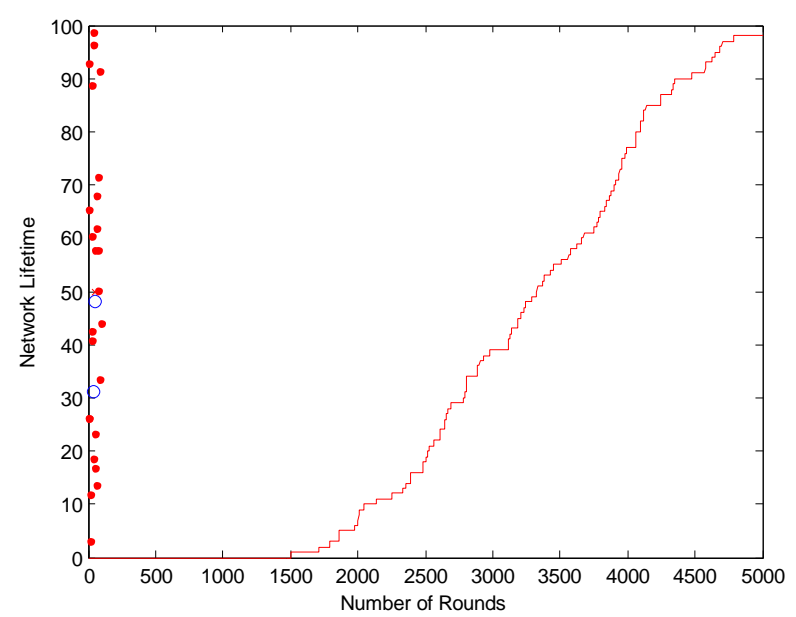

Fig7: Network lifetime of sensor nodes in Fuzzy logic based DDEEC

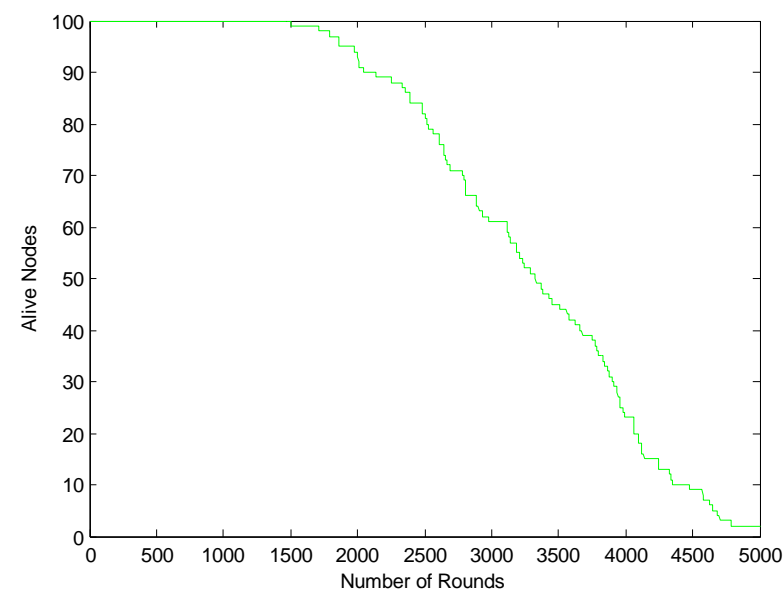

Fig8: No. of alive sensor nodes with different rounds in Fuzzy logic based DDEEC

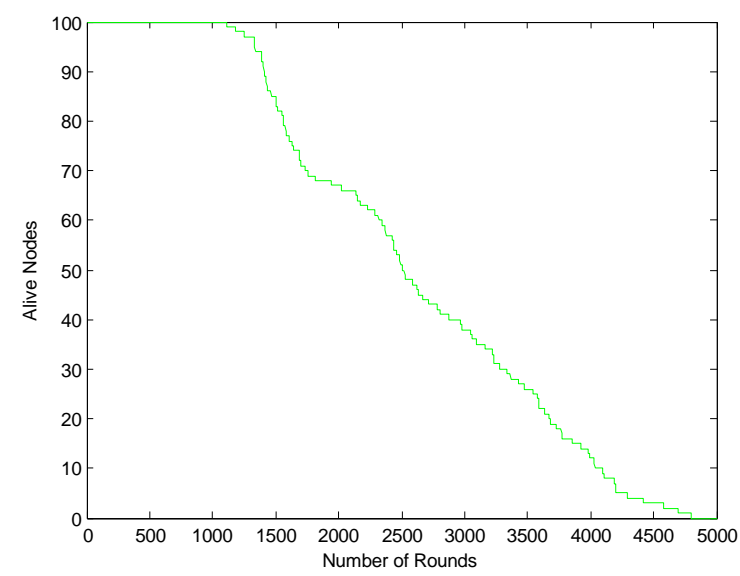

Fig7: No. of alive sensor nodes with different rounds in PSO based DDEEC 


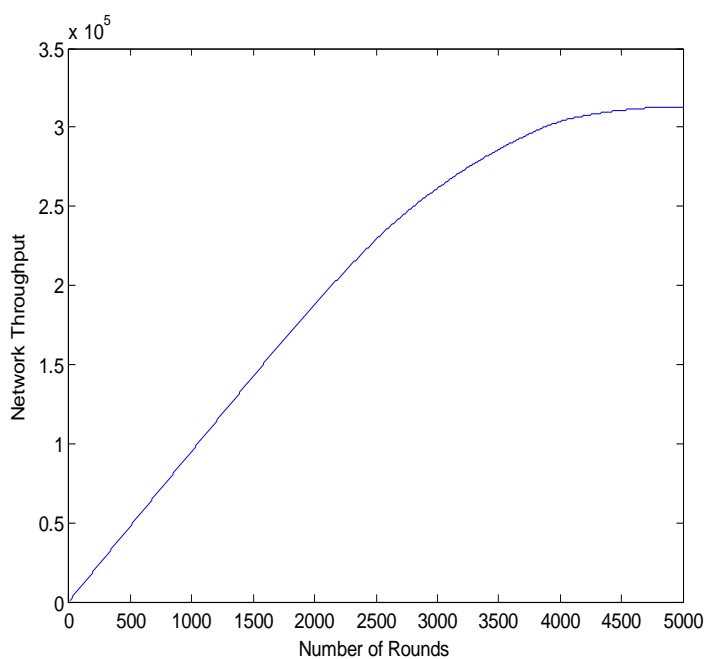

Fig 8: Network throughput in Fuzzy logic based DDEEC at different rounds

Here, 100 nodes are placed randomly between $(x=0, y=0)$ and $(x=100, y=100)$. The location of BS is $(50,50)$.In this first part, results are collected when pso concept is implemented in DDEEC protocol. This algorithm have optimized the selection of cluster-heads using fitness function of Particle Swarm Optimization algorithm is used to maximize life-cycle of the network. The sink and source nodes communicate with each other and maintain the routing with enough residual energy so that clustered structure may claim for maximum lifetime. In this algorithm we have different rounds for the cluster head selection based on threshold energy levels of the given nodes. In DDEEC, no of dead nodes starts occurring at $900^{\mathrm{TH}}$ rounds and this number is gradually increasing and all nodes become dead at 3200 round but in PSO based DDEEC no of dead nodes starts around 1235 round and it goes to $4850^{\mathrm{TH}}$ round when complete network is totally dead. Then we implemented genetic algorithm approach in DDEEC protocol to enhance the life span of the network and increase the throughput but network life time is not extended and it goes up to the 4300 rounds. The performance of the proposed algorithm has been compared with DDEEC PSO-DDEEC. Lastly we propose Fuzzy Logic approach in DDEEC in which fuzzy rule base is designed having 9 rules on the basis of residual energy and minimum distance from the base station. In FL-DDEEC, first node dies at around $1500^{\text {th }}$ round as compared to PSODDEEC \&DDEEC. Network throughput is maximized in this network and achieved highest packet flow from the cluster heads to the base station. The table 1 and 2 shows the comparison of the techniques in terms of the dead node count and the no. of packets transmitted in the networkHere, it is observed that if FND is considered, about $11.5 \%$ round is increased by the FL-DDEEC algorithm but in PSO-DDEEC and GA- DDEEC around 6\% round is increased as compared to original DDEEC . So, comparing to DDEEC, the total lifetime of the WSN can be increased by $11.5 \%$ with this proposed algorithm, which is certainly more energy efficient. Table 1 and table2 depict that this fuzzy based algorithm has better performance than DDEEC in terms of FND (First Node Dies), HNA (Half of Nodes Alive) and LND (Last Node Dies). The amount of data received by the BS is shown in Fig. 2(b). It is noticeable from table 2. that Comparing to DDEEC, about $43.5 \%$ more data can be processed by Fuzzy Logic clustering technique, if packet transmission is considered; whereas about $24.4 \%$ and $22.8 \%$ more data are received by the BS during PSO and GA technique respectively.
Table: Dead Nodes count in the clustered network

\begin{tabular}{|l|l|l|l|}
\hline Dead nodes & FND & TND & $\begin{array}{l}\text { All Nodes } \\
\text { Dead }\end{array}$ \\
\hline DDEEC & 897 & 1098 & 3200 \\
\hline PSO-DDEEC & 1238 & 1459 & 4841 \\
\hline FL-DDEEC & 1456 & 2175 & 5000 \\
\hline
\end{tabular}

Table1: Number of Packets transmitted to the base station in the clustered network

\begin{tabular}{|l|c|c|}
\hline \multicolumn{1}{|c|}{ Protocols } & Pkt to BS & Count Of CH \\
\hline DDEEC & 79617 & 65 \\
\hline PSO-DDEEC & 150754 & 56 \\
\hline FL-DDEEC & 294805 & 17 \\
\hline
\end{tabular}

\section{CONCLUSION}

Issues of node deployment, localization, energy-aware clustering, and data-aggregation are often formulated as optimization problems. FL-DDEEC algorithm is proposed in this paper which optimized the selection of cluster-heads using Fis inference function rule base to achieve maximum life-cycle for a node in a network. The packet transfer rate at base station reaches a high level when the rounds are increasing in FL-DDEEC. The Fuzzy logic based heterogeneous network is more efficient and it maximizes network life. Since the cluster-head is the node responsible for the high energy transmissions to the base station, selecting a single static cluster-head would have drained out the power drastically; our approach is to rotate the cluster-head on the basis of the defuzzified chance value. Thus, all the nodes are compared on the basis of chances and the node with the maximum chance is then elected as the cluster-head. The primary idea was to find means as how to incorporate different parameters that affects the node election criterion with the provision for rotating the cluster-head to conserve energy.

\section{REFERENCES}

[1] N. Vlajic and D. Xia Wireless Sensor Networks: To Cluster or Not To Cluster? WoWMoM'06, 2006.

[2] Vivek Katiyar, Narottam Chand, Surender Soni, Clustering Algorithms for Heterogeneous Wireless Sensor Network: A Survey International Journal of Applied Engineering Research, DINDIGUL Volume 1, No 2, 2010 .

[3] S. Lindsey, C.S. Raghavenda, PEGASIS: "power efficient gathering in sensor information systems", in: Proceeding of the IEEE Aerospace Conference, Big Sky, Montana, March 2002.

[4] O. Younis, S. Fahmy, HEED: "A hybrid, energyefficient, distributed clustering approach for ad hoc sensor networks", IEEE Transactions on Mobile Computing 3 (4) (2004) 660-669.

[5] G. Smaragdakis, I. Matta, A. Bestavros, "SEP: A Stable Election Protocol for clustered heterogeneous wireless 
sensor network", in: Second International Workshop on Sensor and Actor Network Protocols and Applications (SANPA 2004), 2004.

[6] E. Mamdani, S. Assilian, An Experiment in Linguistic Synthesis with a Fuzzy Logic Controller, International Journal of Man-Machine Studies, Vol. 7, Isuue1, pp. 113,1975 .

[7] L. Qing, Q. Zhu, M. Wang, "Design of a distributed energy-efficient clustering algorithm for heterogeneous wireless sensor network", ELSEVIER, Computer Communications 29, 2006, pp 2230- 2237.

[8] Elbhiri, B., Saadane, R., El Fkihi, S., Aboutajdine, D. "Developed Distributed Energy-Efficient Clustering (DDEEC) for heterogeneous wireless sensor networks", in: 5th International Symposium on I/V Communications and Mobile Network (ISVC), 2010.

[9] Parul Saini, Ajay.K.Sharma, "E-DEEC- Enhanced Distributed Energy Efficient Clustering Scheme for heterogeneous WSN", in: 2010 1st International Conference on Parallel, Distributed and Grid Computing (PDGC - 2010).

[10] DuarteMelo, E.J. and Mingyan, L., "Analysis of energy consumption and lifetime of heterogeneous wireless sensor networks", Global Telecommunications Conference. GLOBECOM '02. IEEE, 1: pp 2125, 2002.
[11] V. Katiyar, N. Chand, S. Soni, "A Survey on Clustering algorithms for Heterogeneous Wireless Sensor Networks" Int. J. Advanced Networking and Applications Vol. 02, Issue: 04, pp. 745-754, 2011.

[12] D. Kumar, T. C. Aseri and R. B. Patel, "EEHC: Energy efficient heterogeneous clustered scheme for wireless sensor networks," Computer Communications, Vol. 32 2009, pp. 662-667.

[13] C. Duan and H. Fan, "A Distributed Energy Balance Clustering Protocol for Heterogeneous Wireless Sensor Networks," Wireless Communications, Networking and Mobile Computing, 2007. WiCom 2007. Internationa Conference on, vol., no., 21-25 Sept. 2007, pp. 24692473.

[14] Jun Wang, Xuegang Zhu1, Yong Cheng and Yongsheng Zhu, "A Distributed, Hybrid Energy-Efficient Clustering Protocol for Heterogeneous Wireless Sensor Network", International Journal of Grid and Distributed Computing, Vol. 6, No. 4, August, 2013.

[15] B. Elbhiri, R. Saadane and D. Aboutajdine, "Stochastic Distributed Energy-Efficient Clustering (SDEEC) for heterogeneous wireless sensor networks," ICGST-CNIR Journal, Volume 9, Issue 2, December 2009, pp.11-17. 